ÁREAS NATURALES PROTEGIDAS SCRIPTA, Año 2, Número 1, Enero-Junio de 2016, es una publicación científica digital de periodicidad semestral iniciativa de la Red de Áreas Naturales Protegidas (RENANP) de las Redes Temáticas de CONACyT. Editor en Jefe responsable Dr. Alfredo Ortega-Rubio. Reservas de Derechos al Uso Exclusivo No. 04-2015-071509261100- 203; ISSN: en trámite. Responsable de la última actualización de este número, Dr. Alfredo OrtegaRubio, Av. Instituto Politécnico Nacional 195, La Paz, Baja California Sur, C. P. 23096, Tel (612) 1238484, fecha de la última modificación 30 Junio 2016. Las opiniones expresadas por los autores no necesariamente reflejan la postura de los editores de la publicación. Queda estrictamente prohibida la reproducción total o parcial de los contenidos e imágenes de esta publicación sin previa autorización de los autores de este número de ÁREAS NATURALES PROTEGIDAS SCRIPTA.

Con deferente gratitud ÁREAS NATURALES PROTEGIDAS SCRIPTA reconoce la colaboración de Lic. Gerardo R. Hernández García en la edición gráfica editorial para esta revista y de la M. en C. Diana Dorantes en la revisión del idioma Inglés. Fotografía de la Portada: Daniel Torres-Orozco. 
Protected Natural Areas National Systems in Latin America; the case of Cuba, Uruguay, and México

\title{
Sistemas nacionales de áreas protegidas en América Latina; los casos de Cuba, Uruguay y México
}

\author{
Nathalie Goldberg ${ }^{1}$, Hakna Ferro-Azcona ${ }^{2}$, Alejandro Espinoza-Tenorio ${ }^{2, *}$, \\ Alejandro Ortega-Argueta², M. Azahara Mesa-Jurado², Everardo Barba-Macías².
}

\section{Abstract}

The implementation of protected areas (PA) in Latin America has followed a complex and challenging path due to the diversity of institutional arrangements, legal framework, and national capacities. Because of this context variation, PA National Systems operate under different management schemes. In this study we selected three Latin American countries to analyze how institutional, political and ecological contexts have influenced the PA National Systems of the region. We highlight the most significant developments in Cuba, México, and Uruguay in their PA National Systems, and governmental challenges facing their administration and operation. Finally, we identified the most important issues on which research should provide new knowledge bases not only from an ecological approach, but also a multidisciplinary one, as a way to facilitate the support for improvements on PA regional management. This study provides a comparative overview on Latin America, offering a glimpse of the state of knowledge and management issues, as a way to advance in biodiversity conservation and resource management linked to the development of scientific disciplines.

Key words: Protected Area. Latin America. Sustainable Management.

\footnotetext{
${ }^{1}$ Maestranda en Manejo Costero Integrado, MCISur - U de la R, Uruguay.

${ }^{2}$ Grupo de Manejo Sustentable de Cuencas y Zona Costera (CYZCO), ECOSUR-Unidad Villahermosa

*Autor de correspondencia
} 


\section{Resumen}

La implementación de las áreas protegidas (AP) en América Latina ha seguido un camino complejo y desafiante debido a la diversidad de esquemas institucionales, marcos legales y capacidades nacionales. Debido a esta variación de contextos, los Sistemas Nacionales de AP (SNAP) operan bajo diferentes esquemas de gestión. En el presente estudio se seleccionaron tres países latinoamericanos para analizar los factores institucionales, políticos y ecológicos que influyen en los SNAP de la región. Se destacan los avances más significativos de Cuba, México y Uruguay en el diseño de los SNAP, y los desafíos que han encontrado los gobiernos para su administración y operación. Finalmente, se identificaron aquellos temas más importantes en los que la esfera académica debe aportar nuevas bases de conocimiento no sólo desde un enfoque ecológico, sino también multidisciplinario que facilite el sustento para mejoras en la gestión regional de AP. Este estudio aporta un panorama comparativo en Latinoamérica que permite vislumbrar el estado del conocimiento y los aspectos de gestión, como una forma de avanzar en el conocimiento científico en las disciplinas de conservación de la biodiversidad y del manejo de recursos ligado al desarrollo.

Palabras clave: Área Protegida. América Latina. Manejo sustentable.

\section{Introducción}

1 Las Áreas Protegidas (AP) son el principal instrumento internacional para la conservación in situ del patrimonio natural (Green y Paine, 1997). Actualmente el 14\% de la superficie terrestre del planeta está protegido bajo este esquema (IUCN, 2012). América Latina no ha sido la excepción, y los gobiernos de cada país han realizado grandes esfuerzos encaminados a diseñar, implementar y administrar sus AP (De la Maza et al., 2003).

Pese a que América Latina es una región que ha recibido apoyo de progamas internacionales de conservación con enfoques similares (Bruner, et al., 2004), los contextos en que se establecieron las AP fueron diferentes y la implementación ha seguido un camino complejo que varía de acuerdo a las particularidades políticas, sociales y ecológicas de cada región (Gómez-Pompa y Kaus, 1999; Guarderas et al., 2008). Estas diferencias particulares de contextos internacionales en el manejo de las AP ha recibido muy poca atención en investigación, sobre todo desde una perspectiva comparativa. De este modo, en el presente artículo analizamos los Sistemas Nacionales de AP (SNAP) de tres países latinoamericanos para identificar cómo sus gobiernos han adoptado este 
instrumento de conservación bajo los lineamientos de la Convención de la Diversidad Biológica.

Un análisis comparativo entre el mar Caribe (Cuba), Norte y Centroamérica (México) y Sudamérica (Uruguay), que poseen como elemento común la existencia de un SNAP para el manejo y conservación del patrimonio biológico (Salas et al., 2007), aporta un panorama de la gestión de las AP en Latinoamérica que permite vislumbrar el estado del conocimiento y aspectos de gestión, como una forma de avanzar en el conocimiento científico en las disciplinas de conservación de la biodiversidad y el manejo de recursos.

\subsection{Tres contextos latinoamericanos}

América Latina es una región con muchos aspectos históricos y culturales en común, pero también con profundas diferencias. Cuba, México y Uruguay representan esta heterogeneidad, pues los tres son muy distintos en diversos aspectos (Figura 1; Tabla I).

Socioeconómicamente, la República de Cuba es un Estado socialista que emergió en el siglo XX, donde la mayoría de los medios de producción y la fuerza de trabajo están bajo el control directo del gobierno, quien ha impulsado notables avances en educación y salud. La actividad agrícola es central en su economía y fundamental para asegurar la autosuficiencia alimentaria, frente a los desafíos impuestos por el bloqueo económico de las últimas décadas (Elbers, 2011). Cuba destaca además como un centro de evolución biológica en el mar Caribe, pues es un archipiélago integrado por una isla principal y cerca de 1,600 isletas y cayos (CNAP, 2013).

Esta complejidad insular permite la presencia de una enorme variedad de ecosistemas costeros, en especial comunidades de manglar, arrecifes de coral y pastos marinos, convirtiendo a Cuba en uno de los países latinoamericanos con mayores reservas de este ecosistema (Elbers, 2011). Además, debido a su aislamiento geográfico, la complejidad y heterogeneidad geológica, tipos de suelo, altitud y clima (Vales et al., 1998), el país posee una diversidad de ecosistemas interiores que se caracterizan por tener un alto nivel de endemismo (Berovides y Gerhartz, 2007; CNAP, 2013) y una alta diversidad faunística (González Alonso, 2012; CITMA, 2014). El inventario de la flora cubana está estimado en 7,500 taxones (González-Torres et al., 2013).

La República Oriental del Uruguay también es un Estado relativamente joven (1830) que tiene una sólida tradición cívica y política, con los últimos treinta años de gran apoyo a la democracia. Su economía se basa en gran parte, en la exportación de materias primas y productos derivados del sector agropecuario, pues la ganadería ha desempeñado históricamente un rol predominante (GEF y PNUD, 2007; DINAMA, 2014). El territorio de Uruguay constituye un ecotono de importancia 
global para la biodiversidad, siendo también una de las áreas de mayor riqueza de especies gramíneas en el mundo. Los pastizales dominan el 70\% del territorio y forman parte del bioma pastizal que se encuentra mundialmente amenazado (Cracco et al., 2005). Los paisajes naturales uruguayos se caracterizan por presentar un mosaico de ecosistemas de bosques, humedales y ecosistemas costeros, además de una densa red hidrográfica formada por numerosos cursos de agua de caudal casi permanente (GEF y PNUD, 2007). Los bosques nativos ocupan un $4 \%$ del territorio con seis tipos de formaciones boscosas (SNAP, 2009b) y se han identificado 3,450 especies nativas (Soutullo et al., 2013; DINAMA, 2014).

El caso de los Estados Unidos Mexicanos es dispar, pues es la segunda economía más grande Fig. 1. Países de estudio: Cuba, México y Uruguay y la distribución de sus Áreas protegidas.
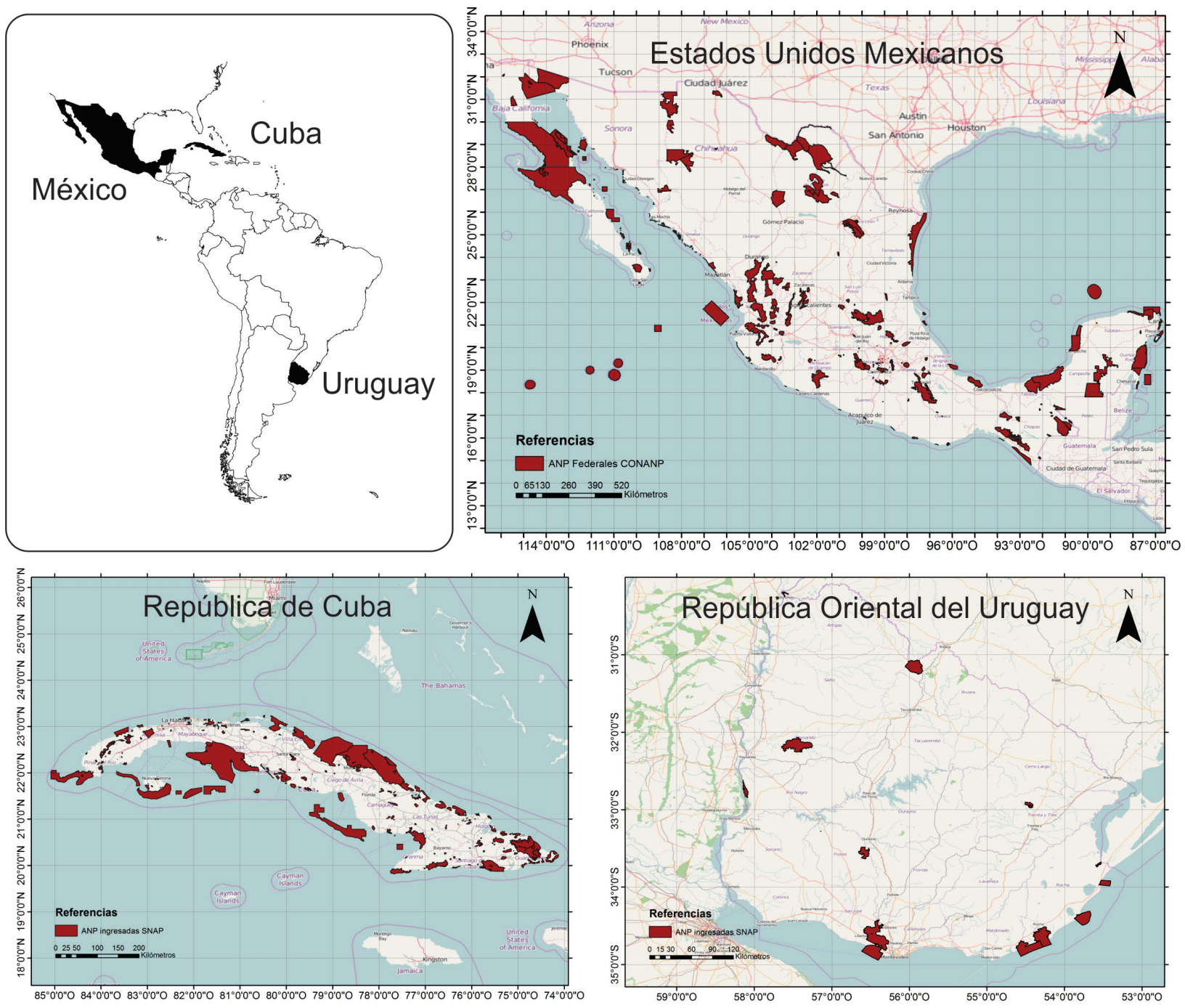

Fuentes: CONANP México (http://www.conanp.gob.mx); SNAP Uruguay (http://www.mvotma.gub.uy/snap.html); SNAP Cuba (http://www.snap.cu); y https:/ / www.google.com.uy/maps/ (Consultado 20/02/2016) 
de Latinoamérica, pero mantiene contrastantes niveles económicos. Socialmente posee una importante diversidad cultural, que se ve reflejada en sus múltiples etnias, religiones, lenguas y tradiciones (CONABIO, 2009). Biológicamente, México destaca por ser una nación megadiversa, pues alberga entre el 10\% y 12\% de la biodiversidad mundial; se ubica entre los dos países con mayor número de ecosistemas y los cinco con mayor diversidad de especies en el mundo (Bellon et al., 2009; Jimenez Sierra et al., 2014). Respecto al ambiente marino, se encuentra entre las influencias oceánicas del Atlántico centro-occidental y el Pacífico centro-oriental, lo que explica su enorme diversidad de especies y ecosistemas marinos (CONABIO, 2009).

Tabla I: Indicadores socioeconómicos.

\begin{tabular}{|c|c|c|c|c|c|c|c|}
\hline & $\begin{array}{l}\text { Sistema } \\
\text { político }\end{array}$ & $\begin{array}{c}\text { Superficie total } \\
\left(\mathrm{km}^{2} \text { - terrestre }+\right. \\
\text { aguas } \\
\text { jurisdiccionales })\end{array}$ & $\begin{array}{l}\text { Población } \\
\text { censo } \\
\text { (miles } \\
\text { habitantes) }\end{array}$ & $\begin{array}{l}\text { Población } \\
\text { estimada } \\
\text { 2015-2020 } \\
\text { (miles } \\
\text { habitantes) }\end{array}$ & $\begin{array}{l}\text { Tasa anual } \\
\text { crecimiento } \\
\text { urbana (\%) }\end{array}$ & $\begin{array}{c}\text { Tasa } \\
\text { alfabetismo } \\
15 \text { a } \\
24 \text { años (\%) }\end{array}$ & $\begin{array}{l}\text { Población } \\
\text { urbana } \\
\text { con agua } \\
\text { potable }(\%)\end{array}$ \\
\hline Cuba & $\begin{array}{l}\text { Estado } \\
\text { socialista }\end{array}$ & 109,884 & 11,210 & 11,281 & 0.2 & 100 & 96.3 \\
\hline México & $\begin{array}{l}\text { República } \\
\text { democrática } \\
\text { representativa } \\
\text { y federal }\end{array}$ & $5,114,295$ & 112,337 & 121,835 & 1.1 & 98.9 & 96.1 \\
\hline \multirow[t]{2}{*}{ Uruguay } & $\begin{array}{c}\text { República } \\
\text { democrática con } \\
\text { un Estado } \\
\text { de carácter mixto } \\
\text { y presidencialista }\end{array}$ & 318,413 & 3,286 & 3,430 & 0.4 & 99.0 & 99.9 \\
\hline & $\begin{array}{l}\text { Tasa de pobreza } \\
\text { urbana }(\%)\end{array}$ & $\begin{array}{c}\text { Tasa de indigencia } \\
\text { urbana }(\%)\end{array}$ & $\begin{array}{c}\text { Tasa de } \\
\text { desempleo }\end{array}$ & IDH (2013) & $\begin{array}{c}\text { Gasto } \\
\text { público } \\
\text { social (\%PBI) }\end{array}$ & $\begin{array}{l}\text { Gasto público } \\
\text { educación } \\
(\% \mathrm{PBI})\end{array}$ & $\begin{array}{l}\text { Gasto } \\
\text { público } \\
\text { salud } \\
(\% \mathrm{PBI})\end{array}$ \\
\hline Cuba & $\mathrm{s} / \mathrm{d}$ & $\mathrm{s} / \mathrm{d}$ & 3.3 & $\begin{array}{c}\text { (44) muy } \\
\text { elevado } 0.815\end{array}$ & 36.5 & 12.8 & 8.6 \\
\hline México & 33.2 & 9.8 & 6.1 & $\begin{array}{l}\text { (71) elevado } \\
0.756\end{array}$ & 10.7 & 5.2 & 6.2 \\
\hline Uruguay & 6.1 & 1.2 & 6.9 & $\begin{array}{l}\text { (50) elevado } \\
0.790\end{array}$ & 24.1 & 4.5 & 9.0 \\
\hline
\end{tabular}

Fuentes: Base de datos y publicaciones estadísticas de la CEPAL. http:/ / estadisticas.cepal.org/ consultado 2/2015. Naciones Unidas 2000-2015. PNUD Informe sobre desarrollo humano 2014 


\section{Origen de los SNAP}

La historia moderna de las AP en la región se ha dividido en tres etapas (Tabla II).

\subsection{Formativa; esfuerzos pioneros}

Durante la primera parte de esta etapa precursora, en México se establece oficialmente el Parque del Desierto de los Leones (1917) y, en la década de los treintas, el primer sistema de parques nacionales. En esa misma etapa, Cuba instauró el primer territorio de conservación (Parque Nacional Sierra Cristal), y Uruguay sus parques nacionales, que paradójicamente, no eran áreas de preservación de ambientes naturales, sino zonas de recreación de oportunidad (donación, remanente de expropiación, interés histórico y áreas fiscales) donde los ambientes naturales eran sustituidos por forestación con especies exóticas (Tejera, 2006).

Hacia el final de esta etapa, después de la Segunda Guerra Mundial hasta los años setentas, la escena internacional estuvo dominada por la creación de instituciones con alcance global (ej. UICN, 1948; WWF, 1961; PNUD y PNUMA, 1973). Estas instituciones revisan la situación de los países sugiriendo líneas de política ambiental, a la vez que se conforman diversos fondos de ayuda para financiar la adopción de los modelos que promueven (Tejera, 2006). En ese momento, las AP de México pasaron por varios cambios administrativos, mientras las de Cuba se incrementaron, pues en el año 1959 se crearon nueve Parques Nacionales y entre 1960-1970 las primeras Reservas Naturales (CNAP, 2013).

\subsection{Adopción; Programas internacionales}

Las políticas de conservación territoriales tomaron mayor relevancia en la década de los setentas con el establecimiento de la Convención Ramsar (1971), la Convención del Patrimonio Mundial (1972) y con el surgimiento del concepto de Reserva de la Biosfera a partir del programa El Hombre y la Biosfera (1977). Esto propició un nuevo impulso para el establecimiento de AP con criterios científicos y biológicos, incorporándose una visión más social de la conservación de los ecosistemas. El establecimiento de la red mundial de reservas bajo el auspicio de las Naciones Unidas (PNUMA), supone un hito en la evolución de las ANP (De la Maza et al., 2003). En este contexto, Cuba estableció en 1970 las bases para la creación de un Sistema Nacional de áreas naturales y culturales (FAO, 1974) y en 1976 se creó la Comisión Nacional para la Protección del Medio Ambiente y los Recursos Naturales (COMARNA). Esta última se instituyó para orientar las medidas legislativas para la preservación del medio ambiente y el aprovechamiento racional de los recursos naturales (SNAP, 2015). En 1981 se aprobó la ley para la Protección del Medio Ambiente 
y del Uso Racional de los Recursos Naturales, que agrupó políticas y acciones proporcionando la base legal para la creación de la red nacional de áreas protegidas. Asimismo, fueron declaradas dieciséis nuevas zonas rurales protegidas (CNAP, 2013).

Tabla II: Línea de tiempo de la evolución de los sistemas de Áreas Naturales Protegidas de Cuba, México y Uruguay.

\begin{tabular}{|c|c|c|c|c|}
\hline & 1900 & 1970 & 1990 & 2000 \\
\hline & $\begin{array}{l}\text { Etapa formativa; } \\
\text { esfuerzos pioneros }\end{array}$ & $\begin{array}{l}\text { Adopción; Programas } \\
\text { internacionales. } \\
\text { El Hombre y la Biosfera }\end{array}$ & $\begin{array}{l}\text { Incorporación } \\
\text { formal. Cumbre de } \\
\text { la Tierra }\end{array}$ & $\begin{array}{l}\text { Incorporación formal. } \\
\text { GEF / PNUD }\end{array}$ \\
\hline Cuba & $\begin{array}{l}1930 \text { Primer Parque } \\
\text { Nacional }\end{array}$ & $\begin{array}{l}1970 \text { Visita UICN áreas de } \\
\text { elevado valor natural. } \\
1976 \text { Creación COMARNA. } \\
1980 \text { Ley Protección del Medio } \\
\text { Ambiente y del Uso Racional de } \\
\text { los Recursos Naturales, Empresa } \\
\text { Nacional para la Protección de la } \\
\text { Flora y la Fauna. }\end{array}$ & $\begin{array}{l}\text { 1991-92 CITMA, } \\
\text { Agencia del Medio } \\
\text { Ambiente. }\end{array}$ & $\begin{array}{l}1999 \text { SNAP. } \\
2000 \text { Sitios Ramsar. } \\
2001 \text { Sitio del } \\
\text { Patrimonio Mundial } \\
\text { Natural. } 2003 \text { Primeros } \\
\text { Planes del Sistema. }\end{array}$ \\
\hline
\end{tabular}

\begin{tabular}{|c|c|c|c|c|}
\hline México & $\begin{array}{l}1917 \text { Primer Parque } \\
\text { Nacional. } 1935 \\
\text { Sistema Nacional de } \\
\text { Reservas Forestales y } \\
\text { de Parques Nacionales. }\end{array}$ & $\begin{array}{l}1972 \text { Subsecretaría de } \\
\text { Mejoramiento del Ambiente. } \\
1976 \text { Dirección General de } \\
\text { Ecología Urbana. } \\
1977 \text { Dirección General } \\
\text { de Recreación y Parques } \\
\text { Nacionales. } \\
1978 \text { Dirección General de } \\
\text { Reservas y Áreas de Recreación. } \\
1982 \text { Ley Federal de Protección } \\
\text { al Ambiente. } 1983 \text { Secretaría de } \\
\text { Desarrollo Urbano y Ecología. }\end{array}$ & $\begin{array}{l}1988 \text { LGEEPA. } \\
1988 \text { SINAP. } \\
1992 \text { INE. } \\
1994 \text { SEMARNAP, } \\
\text { CONANP. }\end{array}$ & $\begin{array}{l}2000 \text { Comisión Nacional } \\
\text { para el Conocimiento y } \\
\text { Uso de la Biodiversidad } \\
\text { (CONABIO) y Fondo } \\
\text { Mexicano para la } \\
\text { Conservación de la } \\
\text { Naturaleza (FMCN). }\end{array}$ \\
\hline Uruguay & $\begin{array}{l}\text { 1916-1946 Parques } \\
\text { Nacionales } \\
\text { "recreativos" creados } \\
\text { por el MDN y el } 1935 \\
\text { MGA }\end{array}$ & $\begin{array}{l}\text { 1946-1982 MGA modifica } \\
\text { política de Parques Nacionales } \\
\text { a la protección de ambientes } \\
\text { naturales y biodiversidad. } \\
\text { 1982 Convenio Internacional } \\
\text { sobre las Zonas Húmedas de } \\
\text { Importancia Internacional } \\
\text { (Ramsar 1971) Reserva de } \\
\text { Biosfera Bañados del Este. } 1986 \\
\text { MGAP. }\end{array}$ & $\begin{array}{l}1989 \text { Red de } \\
\text { Cooperación } \\
\text { Técnica en Parques } \\
\text { Nacionales y Áreas } \\
\text { Protegidas. } \\
1990 \text { MVOTMA. } \\
1993 \text { Convenio } \\
\text { Internacional sobre } \\
\text { la Diversidad } \\
\text { Biológica (Río, } \\
\text { 1992). }\end{array}$ & $\begin{array}{l}2000 \text { SNAP bajo la } \\
\text { Dirección Nacional } \\
\text { de Medio Ambiente } \\
\text { (DINAMA). } \\
\text { 2005 Decreto 52/05 } \\
\text { Reglamenta la Ley } \\
\text { 17234 de creación del } \\
\text { SNAP. } \\
\text { 2008 Primer AP } \\
\text { incorporada al SNAP. }\end{array}$ \\
\hline
\end{tabular}

UICN: Unión Internacional para la Conservación de la Naturaleza; Cuba= CITMA: Ministerio de Ciencias, Centro Nacional de Áreas Protegidas; COMARNA: Comisión Nacional para la Protección del Medio Ambiente y los Recursos Naturales; SNAP: Sistema Nacional de Áreas Protegidas; México= CONANP: Comisión Nacional de Áreas Naturales Protegidas; INE (ahora INECC): Instituto Nacional de Ecología; LGEEPA: Ley General del Equilibrio Ecológico y la Protección al Ambiente; SEMARNAP: Secretaría de Medio Ambiente, Recursos Naturales y Pesca; SINAP: Sistema Nacional de Áreas Naturales Protegidas; Uruguay= MGA: Ministerio de Ganadería y Agricultura; MGAP: Ministro de Ganadería Agricultura y Pesca; MVOTMA: Ministerio de Vivienda, Ordenamiento Territorial y Medio Ambiente; MDN: el Ministerio de Defensa Nacional; SNAP: Sistema Nacional de Áreas Protegidas.

En esta etapa, en México se creó la Ley Federal de Protección al Ambiente (1982) que favoreció a las AP, pues sólo en el año 1983 se conformaron catorce Reservas de la Biosfera y otras categorías de manejo para AP. Esto desembocó en la creación del Sistema Nacional de Áreas Naturales Protegidas (SINAP) en 1988 (Melo Gallegos, 2002). 
En Uruguay se introdujeron las pautas internacionales para la conservación de áreas silvestres de valor patrimonial por sus entornos naturales y paisajísticos. Se modificó además la antigua política de Parques Nacionales a la protección de ambientes naturales y biodiversidad, surgiendo así otros Parques Nacionales y Monumentos Naturales. Las AP continuaron bajo la responsabilidad del Ministerio de Defensa Nacional (MDN) y el Ministerio de Ganadería y Agricultura (MGA, ahora MGAP) (Tejera, 2006).

\subsection{Incorporación formal}

La Cumbre de la Tierra (Río de Janeiro, 1992) significó un gran impulso en el fortalecimiento de las políticas nacionales de AP en América Latina, pues se recibió el apoyo económico del Banco Mundial para la creación de políticas nacionales de AP (Espinoza-Tenorio et al., 2011).

La Convención de Diversidad Biológica (CDB) señaló la obligación de los países de elaborar estrategias de conservación in situ (De la Maza et al., 2003), ocasionando que muchos países reforzaran el marco normativo y desarrollaran una plataforma programática alrededor de las AP.

En Cuba, la aparición de nuevas resoluciones e instituciones a inicios de la década de los noventas permitió el establecimiento de treinta y dos nuevas AP, pero sobre todo el inicio del proceso de institucionalización del SNAP. En 1994 fue creada la Empresa Nacional para la Protección de la Flora y la Fauna, entidad que administra la mayor cantidad de AP en el país; se reconocieron por primera vez treinta y cinco AP marinas, y se designaron seis sitios Ramsar. Aparecieron también los primeros planes del SNAP para encaminar las acciones de conservación a través del desarrollo de líneas estratégicas y, tras la creación del Ministerio de Ciencia, Tecnología y Medio Ambiente, se crea el Centro Nacional de Áreas Protegidas (1994). En el año 2008, el SNAP actualizó su marco legal y se aprobaron 103 AP (CNAP, 2013).

En este período, en México se crearon varias dependencias que significarían un mayor apoyo en la gestión de las AP, como la Comisión Nacional de Áreas Naturales Protegidas (CONANP), la Comisión Nacional para el Conocimiento y Uso de la Biodiversidad (CONABIO) y la unidad coordinadora de Áreas Naturales Protegidas (UCANP), encargada de la administración del SINAP (CONANP, 2015b).

En tanto, en Uruguay en el año 2000 se aprobó la Ley 17234 que declara de interés general la creación del SNAP y su regulación por la Dirección Nacional de Medio Ambiente (DINAMA).

Aunque fue hasta el año 2008, a través de un proyecto de fortalecimiento GEF/PNUD, cuando 
se incorporó la primer AP al sistema. En estos últimos años se avanzó en la planificación y gestión de las AP. Actualmente existen trece áreas ingresadas al SNAP y otras siete en proceso de evaluación para su incorporación (MVOTMA, 2015).

\section{Tipos de áreas protegidas}

Muchas de las categorías de AP fueron creadas en América Latina antes de que se establecieran las sugeridas por la International Union for Conservation of Nature (IUCN) (Tabla 1). De esta forma quedan casos en que la misma categoría de la IUCN tiene varias asignaciones a nivel nacional, y otros donde las asignaciones a nivel nacional no tienen correspondencia con las categorías de la IUCN. En la actualidad, entre los tres países suman un total de 407 AP (29,440,977 ha) que están aprobadas o en proceso de ingreso. La categoría más frecuentemente utilizada es la de Parque Nacional (ochenta y tres ANP), aunque la categoría que cubre más superficie es la de Área Protegida Manejada (20,774,955 ha). La categoría que es menos frecuente es la Reserva Natural Estricta; no se encontraron ejemplos de Áreas Naturales Silvestres.

En Cuba, actualmente son 211 áreas; setenta y siete de Significación Nacional y 134 de Significación Local, además se han propuesto ocho Regiones Especiales de Desarrollo Sostenible. Del total identificadas, 103 están aprobadas y 108 sin aprobar (SNAP, 2015). En su conjunto, las áreas propuestas, administradas y aprobadas, representan un potencial cercano al $18.42 \%$ de la superficie terrestre y marina del país (Tabla III). Su sistema contempla ocho categorías de manejo que van desde aquellas de conservación estricta hasta las de múltiple uso. Las reservas florísticas manejadas (categoría IV) y los refugios de fauna (categoría IV) son las más numerosas. El país cuenta con dos Patrimonios Naturales Mundiales, seis sitios Ramsar y seis Reservas de Biosfera,

y tiene identificadas hasta el momento veintiocho Áreas Importantes para la Conservación de las Aves (IBA) (CNAP, 2013; SNAP, 2015).

México cuenta con 176 áreas naturales de carácter federal que cubren más de 25,000,000 ha; aproximadamente el $80 \%$ corresponde a la superficie terrestre y $20 \%$ a zonas marinas. Los parques nacionales constituyen la categoría más numerosa; sin embargo, las Reservas de Biósfera, ocupan más de la mitad de la superficie total protegida. Del total de $176 \mathrm{AP}$, las aprobadas pertenecientes al SINAP son sesenta y un AP, las cuales cubren una superficie de 12,999,101 ha. México tiene además 367 AP estatales, 120 privadas, cuatro públicas, cuarenta y tres comunales y 201 ejidales, que en conjunto representan más de 405,574 ha. (CONANP, 2015). La CONANP administra sesenta y tres sitios Ramsar dentro de AP y atiende setenta y nueve más en otras zonas (CONANP, 2014b). 
Tabla III. Tipos de áreas protegidas de acuerdo a la similitud de sus objetivos con las categorías de la UICN.

\begin{tabular}{|c|c|c|c|c|c|c|}
\hline $\begin{array}{l}\text { Categorías de } \\
\text { UICN }\end{array}$ & CUBA & S (ha / \%) & MÉXICO & S (ha/ \%) & URUGUAY & S (ha/ \%) \\
\hline $\begin{array}{c}\text { I. Reserva } \\
\text { Natural Estricta }\end{array}$ & $\begin{array}{c}\text { Reserva Natural } \\
\text { (4) }\end{array}$ & $4,358 / 0.02$ & - & - & $\begin{array}{c}\text { Sitios de } \\
\text { protección (0) }\end{array}$ & 0 \\
\hline $\begin{array}{l}\text { I. Área Natural } \\
\text { Silvestre }\end{array}$ & - & - & - & - & - & - \\
\hline $\begin{array}{l}\text { II. Parque } \\
\text { Nacional }\end{array}$ & $\begin{array}{c}\text { Parque Nacional } \\
\text { (14) }\end{array}$ & 944,778 / 5.26 & $\begin{array}{c}\text { Parque Nacional } \\
(66)\end{array}$ & $\begin{array}{c}1,411,319 / \\
0.27\end{array}$ & $\begin{array}{c}\text { Parque } \\
\text { Nacional (3) }\end{array}$ & 33,120 / 0.1 \\
\hline $\begin{array}{l}\text { III. Monumento } \\
\text { Natural }\end{array}$ & $\begin{array}{c}\text { Elemento } \\
\text { Natural } \\
\text { Destacado (33) }\end{array}$ & 142,724 / 0.79 & $\begin{array}{l}\text { Monumento } \\
\text { Natural (5) }\end{array}$ & 16,269 / 0 & $\begin{array}{c}\text { Monumento } \\
\text { Natural (1) }\end{array}$ & $17 / 0$ \\
\hline $\begin{array}{l}\text { IV. Áreas de } \\
\text { Manejo de } \\
\text { Hábitats y/o } \\
\text { Especies }\end{array}$ & $\begin{array}{c}\text { Refugio de } \\
\text { Fauna, Reserva } \\
\text { Florística } \\
\text { Manejada (41) }\end{array}$ & $80,294 / 0.45$ & - & - & $\begin{array}{c}\text { Áreas de } \\
\text { Manejo de } \\
\text { Hábitats y/o } \\
\text { Especies (3) }\end{array}$ & 47,125 / 0.15 \\
\hline $\begin{array}{c}\text { V. Paisaje } \\
\text { Terrestre y } \\
\text { Marino } \\
\text { Protegido }\end{array}$ & $\begin{array}{l}\text { Paisaje Natural } \\
\text { Protegido (24) }\end{array}$ & 44,623 / 0.25 & - & - & $\begin{array}{c}\text { Paisaje } \\
\text { Protegido (4) }\end{array}$ & 80,166 / 0.25 \\
\hline \multirow[t]{2}{*}{$\begin{array}{l}\text { VI. Área } \\
\text { Protegida } \\
\text { Manejada }\end{array}$} & $\begin{array}{l}\text { Área Protegida } \\
\text { de Recursos } \\
\text { Manejados (18) }\end{array}$ & $\begin{array}{c}1,130,483 / \\
6.29\end{array}$ & $\begin{array}{c}\text { Área de } \\
\text { Protección de } \\
\text { Flora y Fauna } \\
\quad(38)\end{array}$ & $\begin{array}{c}6,786,837 \\
/ 1.33\end{array}$ & $\begin{array}{c}\text { Área Protegida } \\
\text { con } \\
\text { Recursos } \\
\text { Manejados (2) }\end{array}$ & $\begin{array}{c}106,486 / \\
0.33\end{array}$ \\
\hline & & & $\begin{array}{c}\text { Reserva de la } \\
\text { Biosfera (41) }\end{array}$ & $\begin{array}{c}12,751,149 \\
\quad / 2.49\end{array}$ & & \\
\hline \multirow[t]{2}{*}{ Otras } & $\begin{array}{l}\text { Refugio de } \\
\text { Fauna (45) }\end{array}$ & $775,730 / 4.32$ & $\begin{array}{c}\text { Área de } \\
\text { Protección } \\
\text { de Recursos } \\
\text { Naturales (8) }\end{array}$ & $\begin{array}{c}4,503,345 \\
/ 0.88\end{array}$ & $\begin{array}{c}\text { Propuestas } \\
\text { para su ingreso } \\
\text { (7) }\end{array}$ & $\begin{array}{c}244,713 / \\
0.76\end{array}$ \\
\hline & $\begin{array}{c}\text { Reserva } \\
\text { Ecológica (32) }\end{array}$ & 189,109 / 1.05 & Santuarios (18) & $\begin{array}{c}148332 / \\
0.03\end{array}$ & & \\
\hline TOTAL & 211 & $\begin{array}{c}3,312,099 / \\
18.42\end{array}$ & 176 & $\begin{array}{c}25,617,251 \\
/ 5\end{array}$ & 20 & $511,627 / 1.6$ \\
\hline \multicolumn{7}{|c|}{$\begin{array}{l}\mathrm{N}=\text { Número de Areas Protegidas; S=Superficie (ha/\% territorio nacional) Para la elaboración de la Tabla III se ha considerado } \\
\text { el conjunto de áreas aprobadas y propuestas (en proceso de aprobación). Los datos de AP en proceso de ingreso en Cuba } \\
\text { y México están distribuidos en las diferentes categorías, en Uruguay están separados por desconocerse a qué categoría } \\
\text { ingresarán. En los cálculos de \% de superficie del territorio nacional, se consideróla superficie total, producto de la sumatoria } \\
\text { de la terrestre, las insulares y las aguas jurisdiccionales. Fuente: CONANP http://www.conanp.gob.mx/que_hacemos/ } \\
\text { fecha: 5/2/2015 http://www.mvotma.gub.uy/snap.html fecha: } 5 / 2 / 2015 \text {, http://www.mvotma.gub.uy/sala-de-prensa/ } \\
\text { noticias/item/10007192-siete-anos-del-snap.html consultado } 1 / 10 / 2015 \text { Base de datos mundial de áreas protegidas (WDPA) } \\
\text { http://www.protectedplanet.net/ consultado 9/2/2015; https://www.iucn.org/es/sobre/union/secretaria/oficinas/ } \\
\text { sudamerica/sur_trabajo/sur_aprotegidas/ap_categorias.cfm consultado 2/10/2015 }\end{array}$} \\
\hline
\end{tabular}

En Uruguay, el territorio bajo distintas formas de protección de la biodiversidad se ubica en el entorno del 8\% de la superficie terrestre y marina del país. El sistema reconoce seis categorías de manejo y en los últimos años incorporó formalmente un total de trece AP dentro del SNAP, 
lo que representa un $0.84 \%$ (266,914 ha) de cobertura del territorio nacional (MVOTMA, 2015). Además, el país cuenta con dos sitios Ramsar, dos Reservas de Biosfera UNESCO, veintidós Áreas de Importancia para las Aves IBA y dos Áreas de Aves Endémicas (DINAMA, 2014).

\section{Sistemas de Áreas Naturales Protegidas: descentralización, gestión compartida y financiamiento}

Los tres países presentan diferentes sistemas de organización, unos en pleno crecimiento, como el caso de Cuba y México, y otro incipiente en Uruguay (Tabla IV). Además del financiamiento gubernamental y de los incentivos fiscales, también existen otras fuentes alternativas, tales como la inversión de capital privado, cooperación binacional con otros gobiernos, el cobro del ingreso a las AP, y la cooperación de organismos multilaterales como el GEF, UNESCO, PNUD, BID, PNUMA, pero sobre todo el Banco Mundial (SNAP, 2010; Gobierno de México, 2013).

\subsection{Sistema centralizado cubano}

En Cuba, si bien la coordinación del sistema es una responsabilidad del Centro Nacional de Áreas Protegidas que pertenece al Ministerio de Ciencia Tecnología y Medio Ambiente (CITMA), las AP se encuentran bajo la responsabilidad de distintas entidades.

La institución que administra la mayor cantidad de AP en el país es la Empresa Nacional para la Conservación de la Flora y Fauna, seguida del Ministerio de la Agricultura, y del propio CITMA. Además, se encuentran las Empresas Forestales Integrales y otras entidades provinciales del CITMA. El resto de las AP son administradas por otros organismos como la ONG Fundación Antonio Núñez Jiménez para la Naturaleza y el Hombre, la Sociedad Anónima Gaviota y el Órgano Local del Poder Popular de la Habana (CNAP, 2013). Para asegurar una gestión integral, se ha establecido una Junta de Coordinación del SNAP, la cual funge como Órgano de Dirección Colegiado presidido por el Centro Nacional de Áreas Protegidas (CNAP), con la participación de las principales instituciones que inciden sobre las áreas. Igualmente, el esquema se ha descentralizado a través de la creación de las Juntas Coordinadoras provinciales de AP y de otras juntas especiales, como las que coordinan las áreas con reconocimiento internacional (CNAP, 2013).

El marco legal del SNAP agrupa un conjunto de disposiciones normativas para regular su funcionamiento. Los costos de la administración de AP en Cuba dependen esencialmente del presupuesto del Estado, el cual es la principal fuente de financiamiento a través del Fondo 
Nacional de Desarrollo Forestal (FONADEF) y del Ministerio de la Agricultura. Otros fondos que también contribuyen son el Fondo de Medio Ambiente y los Programas Territoriales, Nacionales y Ramales de Investigación y Desarrollo. Sin embargo, en Cuba el financiamiento internacional constituye una valiosa fuente adicional de recursos que fortalece la gestión y permite atender algunas necesidades puntuales. Algunas de las organizaciones internacionales que han tenido mayor participación en financiamiento son: WWF-Canadá, Programa de Pequeñas Donaciones del Programa de las Naciones Unidas (PPD/PNUD); Fondo Mundial para el Medio Ambiente del Programa de las Naciones Unidas (GEF/PNUD).

\subsection{Sistema federal mexicano}

Tanto la política de conservación a través de AP, como la institución a cargo de su administración en México, se encuentran en proceso de consolidación, específicamente en lo concerniente a la creación y manejo de estas áreas (Gobierno de México, 2013). México cuenta con una instancia del Gobierno Federal en el sector ambiental, rectora de las AP, que se encuentra descentralizada administrativamente, y con instancias paralelas de menor nivel jerárquico que también participan autónomamente en la declaratoria, administración y manejo de las AP, es decir, con un modelo descentralizado. Además del proceso de regionalización de AP que diseñó la CONANP, dividiendo al país en siete zonas (CONANP, 2007), un número creciente de estados y municipios han creado sus propios sistemas de AP (Castaño-Uribe, 2007). Actualmente existe una diversidad de esquemas, entre los que se pueden mencionar: el Corredor Biológico Mesoamericano-México, los sitios Ramsar para la protección de humedales de importancia internacional, las áreas de importancia para la conservación de las aves, las áreas de refugio para la protección de especies acuáticas, los campamentos tortugueros, las servidumbres ecológicas, las áreas comunitarias protegidas, las áreas ejidales, las unidades de manejo de vida silvestre y los sitios sagrados naturales (Elbers, 2011).

Una modalidad de AP especialmente interesante es la denominada Áreas Destinadas Voluntariamente a la Conservación; se trata de espacios naturales de propiedad privada, pública, comunal y ejidal, certificados voluntariamente por sus propietarios como AP. Esta modalidad de conservación ha tenido una amplia aceptación (Gobierno de México, 2013) pues es el resultado de múltiples esfuerzos individuales, aislados y desagregados efectuados por una diversidad de actores sociales (Castaño-Uribe, 2007). 
Tabla IV. Forma de organización de los sistemas de Áreas Protegidas de Cuba, México y Uruguay

\begin{tabular}{|c|c|c|c|}
\hline & Nivel Nacional & $\begin{array}{l}\text { Nivel Provincial/ Estatal / } \\
\text { Departamental }\end{array}$ & Nivel Local \\
\hline Cuba & $\begin{array}{l}\text { Ministerio de Ciencia Tecnología y } \\
\text { Medio Ambiente. } \\
\text { Junta Coordinadora Nacional de } \\
\text { Áreas Protegidas. } \\
\text { CNAP CGB ENPFF, ORASEN. } \\
\text { Ministerio de la Industria } \\
\text { Alimentaria. } \\
\text { CITMA DMA MINFAR. }\end{array}$ & $\begin{array}{l}\text { Junta Coordinadora Provincial de } \\
\text { Âreas Protegidas, conformada por la } \\
\text { Delegación Territorial del CITMA, el } \\
\text { Cuerpo de Guardabosque, la Oficina } \\
\text { Provincial de Inspección Pesquera, } \\
\text { el Servicio Estatal Forestal (SEF), los } \\
\text { establecimientos territoriales de la } \\
\text { Empresa Nacional para la Protección } \\
\text { de la Flora y la Fauna, y los Órganos } \\
\text { Locales del Poder Popular }\end{array}$ & $\begin{array}{l}\text { ANP que poseen administración, } \\
\text { tanto individual (ejercida por una } \\
\text { única persona natural o jurídica) } \\
\text { como coordinadas (ejercida a través } \\
\text { de una Junta de Administración), } \\
\text { compuesta por todas las personas } \\
\text { naturales o jurídicas con intereses } \\
\text { en el ANP. }\end{array}$ \\
\hline México & $\begin{array}{l}\text { SEMARNAT } \\
\text { CONANP SINAP } \\
\text { CONABIO FMCN }\end{array}$ & $\begin{array}{l}\text { A nivel estatal, las ANP están bajo la } \\
\text { administración de Secretarías (de medio } \\
\text { ambiente, recursos naturales, desarrollo } \\
\text { urbano y ecología, etc.) o Institutos (de } \\
\text { ecología, desarrollo sustentable, medio } \\
\text { ambiente, etc.) de los Gobiernos de } \\
\text { los Estados. Algunos cuentan con un } \\
\text { Sistema Estatal de Áreas Naturales }\end{array}$ & $\begin{array}{l}\text { Algunos Municipios han creado } \\
\text { ANP. También están las iniciativas } \\
\text { civiles de conservación voluntaria. } \\
\text { Actualmente existen } 367 \text { Áreas } \\
\text { Destinadas Voluntariamente } \\
\text { comunitarias o ejidales protegidas } \\
\text { y áreas privadas. }\end{array}$ \\
\hline Uruguay & $\begin{array}{l}\text { MVOTMA } \\
\text { DINAMA División de } \\
\text { Biodiversidad y Áreas Protegidas } \\
\text { Departamento de Gestión del } \\
\text { SNAP } \\
\text { DINOT DINAGUA COTAOT } \\
\text { COTAMA CNA } \\
\text { MGAP MDN }\end{array}$ & $\begin{array}{l}\text { A nivel departamental, las instituciones } \\
\text { relacionadas al SNAP son las } \\
\text { Intendencias Departamentales, los } \\
\text { Municipios y la Junta Departamental. } \\
\text { Algunos departamentos se encuentran } \\
\text { creando los Sistemas Departamentales } \\
\text { de ANP }\end{array}$ & $\begin{array}{l}\text { Cada ANP del SNAP cuenta con } \\
\text { una Comisión Asesora Especifica } \\
\text { del Área Protegida (CAE) de acción } \\
\text { local. Integrada por representantes } \\
\text { de los diversos ministerios e } \\
\text { instituciones públicas relacionadas, } \\
\text { y representantes de la sociedad } \\
\text { civil interesados, como ONG, } \\
\text { productores rurales, vecinos de la } \\
\text { zona, etc. }\end{array}$ \\
\hline
\end{tabular}

Fuente: CONANP, México http://www.conanp.gob.mx/; CNAP (2013) Plan del SNAP 2014-2020, Cuba SNAP (2009) Plan de Mediano Plazo 2010-2014, Uruguay; Cuba= CGB: Dirección Nacional del Cuerpo de Guardabosque; CITMA: Ministerio de Ciencia, Tecnología y Medio Ambiente; CNAP: Centro Nacional de Áreas Protegidas; DMA: Dirección de Medio Ambiente; ENPFF: Empresa Nacional para la Protección de la Flora y la Fauna; MINFAR; Ministerio de las Fuerzas Armadas; y ORASEN: Oficina de Regulación Ambiental y de Seguridad Nuclear. México= CONABIO: Comisión Nacional para el Conocimiento y Uso de la Biodiversidad; CONANP: Comisión Nacional de Áreas Naturales Protegidas; FMCN: Fondo Mexicano para la Conservación de la Naturaleza; y SEMARNAT: Secretaría de Medio Ambiente y Recursos Naturales. Uruguay= COTAMA: Comisión Técnica Asesora de Medio Ambiente; COTAOT: Comisión Técnica Asesora de Ordenamiento Territorial; CNA: Comisión Nacional Asesora de Áreas Protegidas; DINAGUA: Dirección Nacional de Aguas; DINAMA: Dirección Nacional de Medio Ambiente; DINOT: Dirección Nacional de Ordenamiento Territorial; MDN: Ministerio de Defensa Nacional; MGAP: Ministerio de Ganadería Agricultura y Pesca; y MVOTMA: Ministerio de Vivienda, Ordenamiento Territorial y Medio Ambiente.

Los programas y acciones que desarrolla la CONANP se han orientado a beneficiar a las comunidades humanas que habitan en las AP, a través de una serie de Programas de Subsidio dirigidos al apoyo a los propietarios, poseedores y usufructuarios del territorio y los recursos naturales. Adicionalmente, con estos programas se busca fortalecer la organización comunitaria, su diversificación productiva y la realización de actividades económicas sustentables que generen ingresos para sus habitantes, muchos de ellos en condiciones de pobreza. Se calculan más de 73,000 personas beneficiadas a través de los subsidios a programas como el Programa de Conservación 
para el Desarrollo Sostenible, el Programa de Vigilancia Comunitaria, y el Programa de Acción para la Conservación de la Especie Vaquita Marina; así como también se brinda apoyo a Proyectos Productivos Alternativos vinculados al cultivo de plantas y alimentos, miel, acuacultura, pesca, turismo integral, café orgánico, y productos reciclables (se registraron 790 Proyectos Productivos Alternativos en 128 ANP) (Gobierno de México, 2013).

El Fondo Mexicano para la Conservación de la Naturaleza $(\mathrm{FMCN})$, que tiene un esquema público-privado, y administra los recursos financieros y técnicos para programas y proyectos de conservación, es quien desde 1997 se encarga del manejo del Fondo para Áreas Naturales Protegidas (FANP). En la última década, el FANP ha cuadruplicado su patrimonio a través de aportaciones del Banco Mundial y donantes adicionales. La mayoría de los recursos se destinan a apoyar Proyectos Innovadores Estratégicos de conservación ejecutados por organizaciones de la sociedad civil mexicana (CONANP, 2014). Bajo distintos esquemas de colaboración internacional, México ha generado espacios de cooperación con varias instituciones y organismos internacionales, entre los que destacan la Agencia Alemana de Cooperación para el Desarrollo, el Banco Alemán de Desarrollo, la Agencia Francesa de Desarrollo y el GEF (Gobierno de México, 2013).

\subsection{Sistema incipiente uruguayo}

El Departamento de Gestión del SNAP se encuentra dentro de la División de Biodiversidad y Áreas Protegidas perteneciente al Ministerio de Vivienda, Ordenamiento Territorial y Medio Ambiente (MVOTMA), quién a través de la Dirección Nacional de Medio Ambiente (DINAMA) tiene competencia para regular las políticas del SNAP. Dentro de las AP ocurre una intersección de jurisdicciones de otras instituciones del gobierno nacional, departamental y local, que tienen diversas competencias en temas de relevancia para el SNAP, y distinto grado de injerencia sobre el mismo territorio.

Además, existen otros actores con un rol fundamental vinculado al manejo de las ANP, hay aproximadamente 70 instituciones de la academia y de la sociedad civil y casi 60 ONG. Estas organizaciones han estado involucradas desarrollando planes de manejo y propuestas para ingreso de AP, actividades relacionadas con la investigación, educación ambiental, asesoramiento en políticas, mediadoras en conflictos, realizando estudios y consultorías ambientales, entre otras (GEF y PNUD, 2007). Como ámbito de participación ciudadana y coordinación interinstitucional, está la Comisión Nacional Asesora (CNA), órgano asesor del MVOTMA, donde se interviene en 
todo lo relativo a la política de AP a nivel nacional, así como el cumplimiento de la ley 17234. Como ámbito de participación de las comunidades locales, en cada ANP hay una Comisión Asesora Específica (CAE), encargada de realizar el seguimiento y control de las AP, y velar por el cumplimiento de los Planes de Manejo.

Durante el siglo XX, a través de diferentes leyes, decretos nacionales o resoluciones municipales con objetivos y características muy diferentes, se les ha otorgado algún tipo de protección legal a veintiséis áreas, que en su mayoría operaban como unidades individuales sin constituir partes de un sistema. En estos siete años de creación del SNAP, a pesar de la baja cobertura de superficie, el porcentaje de paisajes representados supera el 70\% del total del país, y el de ecosistemas y especies prioritarias para la conservación amenazados representado supera el 30\% del total (MVOTMA, 2015). Pero no todas incluyen una muestra representativa de la biodiversidad del país, ni muchos elementos de valor significativo quedan aún fuera de la protección (principalmente praderas y ecosistemas marinos) (GEF y PNUD, 2007; SNAP, 2009b).

En relación a las fuentes de financiamiento del SNAP en Uruguay, la legislación específica prevé varias fuentes: recursos presupuestales, subsidios, proventos, multas y decomisos, creación de impuestos específicos, herencias y donaciones, cobro de tarifas para el ingreso a las AP (Art. 16, Ley 17234). La legislación también contempla el carácter mixto o privado de las administraciones de las AP, lo que incorpora implícitamente la posibilidad de que el SNAP obtenga fuentes privadas de financiamiento. Está previsto que la administración de estos recursos financieros se haga a través del Fondo de Áreas Protegidas, pero aún no se encuentra operando, no ha sido reglamentado, ni capitalizado. Las posibilidades de fuentes de financiamiento que brinda la normativa no han sido explotadas adecuadamente, y las decisiones políticas no han sido adoptadas (SNAP, 2010).

En la actualidad, el SNAP depende en gran medida de los fondos de Cooperación Internacional Multilateral (GEF, PNUD, Cooperación Francesa y Española) y en su mayoría de los recursos del presupuesto quinquenal que se obtienen mediante la Ley de Presupuesto Nacional (SNAP, 2010). El diagnóstico realizado para el Plan de Mediano Plazo del SNAP, estimó que en el año 2008 el gasto directo destinado a la gestión de ANP alcanzó los USD 750,000, de los cuales el 75\% fue aportado por el Estado, el 19\% correspondió mayoritariamente a fondos de cooperación internacional, y el 6\% restante correspondió a ingresos generados en las propias AP que fueron retenidos para gastos de gestión (SNAP, 2009). 
El proceso de implementación delSNAP está en sus etapas iniciales, y aunque desde su formación se ha incrementado la superficie de cobertura, hasta el día de hoy es uno de los países de América Latina que menos porcentaje de superficie protegida tiene incorporada al sistema. En cuanto a las AP que ya han ingresado al SNAP, sólo dos cuentan con Plan de Manejo aprobado, y seis se encuentran en avanzado proceso de elaboración (MVOTMA, 2015). Los planes de manejo se están realizando de formas diversas, con distintos modelos de gobernanza y sistemas de participación para lograr la efectividad del manejo. El Proyecto de Fortalecimiento del Proceso de Implementación del SNAP URU/06/G34, plantea que de esta forma se permitirá probar una variedad de modelos de gobernanza y tipos de manejo bajo diferentes categorías de AP, como parte de una estrategia para desarrollar un SNAP con múltiples actores, y para compartir las responsabilidades y costos del manejo de las AP entre un amplio espectro de instituciones, organizaciones e individuos. Las experiencias demostrativas en las que se está trabajando son: 1) Manejo de un AP (Categoría II UICN) de propiedad pública, involucrando instituciones del gobierno nacional y la participación de las comunidades locales en la gestión y los beneficios derivados; 2) Manejo de un AP costeromarina (Categoría IV UICN) de propiedad pública, involucrando instituciones del gobierno nacional y ONG; 3) Manejo de un AP (Categoría V UICN) de uso múltiple de propiedad públicoprivada, involucrando medianos productores, comunidades locales y gobiernos municipal y nacional.

\section{Consideraciones finales y perspectivas}

La conservación de la biodiversidad es un reto que se intensifica constantemente por las múltiples presiones que se ejerce sobre los ambientes naturales, por lo que es imprescindible fortalecer esfuerzos para aminorarlo. En este sentido, existe consenso mundial respecto a que las AP son un instrumento efectivo para evitar la pérdida de hábitats y conservación de la diversidad biológica y cultural (Green y Paine, 1997), pero no debe ser el único, pues se necesita de toda la gama de instrumentos de protección ambiental para facilitar la protección integral de los ecosistemas mundiales. Los principales desafíos a los que se enfrentan las AP latinoamericanas, son amenazas externas asociadas al cambio climático, al turismo irresponsable, al desarrollo de infraestructura y a las demandas crecientes de uso y explotación de recursos naturales, junto a otras amenazas como la falta de apoyo político y de recursos (IUCN, 2012). Fundamentalmente, las AP se encuentran amenazadas a causa de actividades económicas mal planificadas que responden a intereses de 
corto plazo y ponen en peligro el patrimonio natural, en especial las relativas a infraestructura, la minera, la petrolera, la agroindustrial (principalmente soya y biocombustibles), y la turística (Elbers, 2011). En este sentido, los países latinoamericanos plantean la necesidad de afrontar estrategias que concentren esfuerzos en varios temas, a diferentes niveles (Tabla V).

En Latinoamérica se evidencia que el futuro de las AP estará cada vez más ligado a las agendas político-económicas y a la manera en que la región vaya consolidando sus procesos de integración y perfilando sus modelos de desarrollo (Elbers, 2011). Cuba, México y Uruguay han tenido avances relevantes en la integración a sus políticas ambientales pero, como en muchas partes del mundo, aún tienen grandes retos por enfrentar para la efectiva implementación de sus AP (Chavarría, 1988; Ojeda-Revah y Espejel, 1993).

Una de las principales limitaciones son los insuficientes medios financieros y las capacidades institucionales y humanas con que cuentan los gobiernos para gestionar las AP (Guarderas et al., 2008). Por esta razón, un gran porcentaje de AP existen solamente en el instrumento legal de creación, sin que sus disposiciones lleguen a aplicarse en la realidad (Aguilar-Perera y Schärer, 2006; Castrejón y Charles, 2013). Según el estudio de diagnóstico de AP en Latinoamérica y el Caribe realizado por Castaño-Uribe (2007), la segunda más grande asignación presupuestal del gobierno para las AP de la región, en términos absolutos, es de México (USD 70,442,212), a la que le sigue Cuba con (USD 47,859,800). Pero cuando la asignacion de recursos de la Nación se analiza por unidad de superficie (USD/ha), Cuba se encuentra en una posición privilegiada, en terminos relativos a la superficie protegida. Según el mismo estudio, ambos países presentan las mayores tasas de incremento anual en la asignación nacional de presupuesto para las AP.

Así, en los tres países se ha avanzado en la creación y ampliación de la cobertura protegida, a través de la delimitación e incorporación de diferentes tipos de ANP, algunas similares a las propuestas por la UICN, otras adaptadas completamente a los contextos nacionales como los Refugios de Fauna de Cuba o los Santuarios de México. Es de resaltar también que la categoría menos frecuente es la que procura la menor presencia humana, pues sólo incluye áreas exclusivas para la investigación y el manejo silvestre, lo que se contrapone a la tendencia en el resto del mundo, donde los menos son los Monumentos Naturales, pero coincide con otros países desarrollo al sur de Asia y la parte tropical de África (Green y Paine, 1997). Ni la categoría más frecuente (Parque Nacional), ni la de mayor superficie (Área Protegida Manejada), coinciden con las Áreas de Manejo de Hábitats y/o especies más frecuentemente utilizadas mundialmente. 
Tabla V. Algunos de los retos para las áreas protegidas en América Latina.

\section{Retos}

1. Ampliar el territorio bajo protección.

2. Lograr representatividad de ecosistemas, diversidad biológica, especies endémicas, especies prioritarias para la conservación y especies en peligro de extinción.

3. Restaurar y recuperar ecosistemas degradados.

4. Avanzar en la protección de ecosistemas marinos y ecosistemas sensibles.

5. Lograr conectividad natural entre las AP y paisajes más amplios, que garanticen continuidad ecológica y corredores biológicos.

6. Profundizar y potenciar el papel de las AP en las medidas de mitigación y adaptación al cambio climático.

7. Integración transfronteriza, con enfoques bio-regionales e internacionales de conservación, que estimulen la colaboración entre países.

8. Elaborar Plan Director del SNAP y Planes de Manejo para cada AP.

9. Elaborar ordenamientos ecológicos del territorio en las AP, y en las zonas de amortiguamiento e influencia.

10. Elaborar programas de protección, restauración y recuperación de ecosistemas y especies.

11. Establecer programas de monitoreo y evaluación, así como sistemas de información.

12. Realizar una valoración de bienes y servicios ambientales de las AP.

13. Evaluar la contribución de valores culturales tangibles e intangibles en las AP.

14. Promover actividades productivas sustentables que permitan el uso de recursos naturales de forma adecuada.

15. Desarrollar proyectos con alternativas de usos y actividades para estrechar el vínculo entre la conservación y el desarrollo social.

16. Incorporar a los pobladores de las AP y comunidades adyacentes como agentes sociales de conservación.

17. Generar mecanismos de coordinación interinstitucionals e intersectorial, y buscar la transversalidad de las políticas y acciones que contribuyan al logro de la conservación y sostenibilidad.

18. Garantizar recursos económicos suficientes para cumplir los objetivos establecidos.

19. Desarrollar estrategias innovadoras para obtener financiamiento a largo plazo, y mecanismos alternativos que permitan la sostenibilidad financiera.

20. Promover la aplicación efectiva de las convenciones e iniciativas internacionales CDB, Ramsar, Programa MAB, Agenda XXI, y otros.

21. Descentralizar, establecer sistemas regionales, departamentales, estatales, complementando al sistema nacional, salvaguardando áreas de relevancia local

22. Incentivar la creación de AP privadas e incluir a comunidades locales en la gestión, estímulos fiscales, certificación, y pago de servicios ambientales.

23. Adecuar marcos legales, complementar la legislación existente y desarrollar instrumentos, normas, y reglamentos

24. Fortalecer mecanismos de vigilancia y aplicación legislativa

25. Ampliar espacios de participación, consejos técnicos, órganos de consulta con representación de actores involucrados, academia y organizaciones de la sociedad civil

26. Reforzar procesos participativos, solidarios y equitativos desde la sociedad civil organizada, que involucren comunidades rurales, indígenas, pueblos originarios, propietarios privados, sectores productivos.

27. Promover que la investigación se incorpore en los instrumentos de gestión y en la toma de decisiones

28. Fortalecer la capacidad de administración con recursos humanos suficientes y calificados, equipamiento e infraestructura

29. Crear estrategias de comunicación, educación y conciencia pública

30. Promover en las ANP espacios de aprendizaje in situ para la sociedad en general

Fuente: De la Maza et al., 2003; Castaño-Uribe, 2007; Elbers, 2011. 


\section{Agradecimientos}

Se agradece al Consejo Nacional de Ciencia y Tecnología por la beca otorgada a H. Ferro-Azcona. Asimismo, a El Colegio de la Frontera Sur ECOSUR-Unidad Villahermosa, especialmente al Dr.

E. Barba-Macías y al Dr. A. Espinoza-Tenorio por recibir y acompañar a N. Goldberg en estancia académica. Un especial agradecimiento a M. Hernández-Chávez por su apoyo en la redacción del escrito.

\section{Bibliografía}

Aguilar-Perera, A., \& Schärer, M. V.-P. (2006). Marine protected areas in Puerto Rico: Historical and current perspectives. Ocean Coast. Manag.

Bellon, R., Barrientos-Priego, F., Colunga-Marciamarín, P., Perales, H., Reyes-Aguero, J., Rosales-Serna, R., y otros. (2009). Diversidad y conservación de recursos genéticos en planas cultivadas. En CONABIO, Capital Natural de México, Estado de Conservación y Tendencias de Cambio. (págs. 355-382). DF, México.

Berovides, A. V., \& Gerhartz, J. (2007). Diversidad de la vida y su conservación. La Habana, Cuba: Científico-Técnica.

Bruner, A., Gullison, R., \& Balmford, A. (2004). Financial Costs and Shortfalls of Managing and Expanding Protected-Area Systems in Developing Countries. BioScience.

Castaño-Uribe, C. (2007). Diagnóstico y situación actual de las áreas protegidas en América Latina y el Caribe. Informe Regional Corporación Andina de Fomento . CAF.

Castrejón, M., \& Charles, A. (2013). Improving fisheries co-management through ecosystem-based spatial management: The Galapagos Marine Reserve. . Mar. Policy .

Chavarría, E. (1988). Coastal protected areas in Mexico. Corvaillis, Oregon .

CITMA. (2014). V Informe Nacional al Convenio sobre la Diversidad Biológica. La Habana, Cuba.

CNAP. (2013). Plan del Sistema Nacional de Áreas Protegidas 2014-2020. La Habana, Cuba.

CONABIO. (2009). Capital Natural de México. Conocimiento actual, evaluación y perspectivas de sustentabilidad. México.

CONANP. (2007). Programa Nacional de Áreas Naturales Protegidas 2007-2012. México.: Comisión Nacional de Áreas Naturales Protegidas. 
CONANP. (2014). Fondo para las Áreas Naturales Protegidas. Recuperado el 15 de 04 de 2015, de CONANP: http://www.conanp.gob.mx/acciones/fanp.php

CONANP. (2014b). Estrategia hacia 2040: una orientación para la conservación de las áreas naturales protegidas de México. México: Comisión Nacional de Áreas Naturales Protegidas.

CONANP. (2015). Áreas destinadas voluntariamente a la conservación. . Recuperado el 15 de 04 de 2015, de CONANP: http://www.conanp.gob.mx/que_hacemos/areas_certi.php

CONANP. (2015b). CONANP. Recuperado el 01 de 10 de 2015, de Historia: http:/ / www.conanp. gob.mx/quienes_somos/historia.php

Cracco, M., García, L., González, E., Rodriguez, L., \& Quintillán, A. (2005). Importancia global de la biodiversidad de Uruguay. . Montevideo, Uruguay.

De la Maza, J., Cadena-González, R., \& Pinguerón-Wirz, C. (2003). Estado actual de las áreas naturales protegidas de America Latina y el Caribe.

DINAMA. (2014). V Informe nacional a la conferencia de las partes del Convenio de Diversidad Biológica. Montevideo, Uruguay. : MVOTMA.

Elbers, J. (2011). Las áreas protegidas de América Latina: situación actual y perspectivas para el futuro. . Quito, Ecuador: UICN, Gobierno de España.

Espinoza-Tenorio, A., Espejel, I., Wolff, M., \& Zepeda-Domínguez, J. (2011). Contextual factors influencing sustainable fisheries in Mexico. DF, México.: Mar. Policy.

FAO. (1974). Manejo y desarrollo integral de las áreas naturales y culturales.

GEF; PNUD. (2007). Fortalecimiento del Proceso de Implementación del Sistema Nacional de Áreas Protegidas de Uruguay URU/06/G34. Uruguay.

Gobierno de México. (2013). Programa Nacional de Áreas Protegidas 2014-2018. En G. d. México, Plan Nacional de Desarrollo 2013-2018. México.

Gómez-Pompa, A., \& Kaus, A. (1999). From prehispanic to future conservation alternatives: lessons from Mexico. Proceedings of the National Academy of Sciences.

González Alonso, H. (2012). Aves. En H. González Alonso, L. Rodríguez Schettino, A. Rodríguez, C. Mancina, \& I. Ramos, Libro Rojo de los Vertebrados en Cuba (págs. 207-267). La Habana : Academia .

González-Torres, L., Palmarola, A., \& Barrios, D. (2013). Las 50 plantas más amenazadas de Cuba. Bissea. 
Green, J. M., \& Paine, J. (1997). State of the world's protected areas at the end of the twentieth century. (I. W. Areas, Ed.) Protected areas in the 21st century: from islands to networks, 24-29.

Guarderas, A., Hacker Sally, D., \& Lubchenco, J. (2008). Current status of marine protected areas in latin america and the caribbean. Conserv. Biol.

IUCN. (2009). IUCN. Recuperado el 02 de 10 de 2015, de Categorías de manejo de áreas protegidas de la UICN: https://www.iucn.org/es/sobre/union/secretaria/oficinas/sudamerica/ sur_trabajo/sur_aprotegidas/ap_categorias.cfm

IUCN. (2012). Áreas Protegidas ¿Qué son y para qué sirven? Recuperado el 15 de 04 de 2015, de IUCN: http://www.iucn.org/es/sobre/trabajo/programas/areas_protegidas_/copy_of_ aires_protegees__quest_ce_que_cest__quelle_est_leur_utilite__13012012_1127/.

Jimenez Sierra, C., Sosa Ramirez, J., Cortés-Calva, P., Solís Cámara, A., \& Iñiguez Dávalos L. I. Ortega-Rubio, A. (2014). México país megadiverso y la relevancia de las áreas naturales protegidas. . Investigación y Ciencia de la Universidad Autónoma de Aguascalientes.

Melo Gallegos, C. (2002). Áreas naturales protegidas en México en el Siglo XX. DF, México .

MVOTMA. (2015). MVOTMA. Recuperado el 30 de 09 de 2015, de Noticias. Siete años del SNAP: http://www.mvotma.gub.uy/sala-de-prensa/noticias/item/10007192-siete-anos-delsnap.html

Ojeda-Revah, L., \& Espejel, I. (1993). El sistema de áreas naturales protegidas en la frontera de México y Estados Unidos. Front. Norte.

Rylands, A., \& Brandon, K. (2005). Brazilian Protected Areas. Conservation Biology.

Salas, S., Chuenpagdef, R., Seijo, J., \& Charles, A. (2007). Callenges in the as sessment and management of small-scale fisheries in Latin America and the Caribbean. Fisheries Research.

SNAP. (2009). Plan de Mediano Plazo 2010-2014. Montevideo, Uruguay. .

SNAP. (2009b). Sistema Nacional de Áreas Protegidas de Uruguay. Una construcción colectiva. Uruguay. SNAP. (2010). Entendimiento del marco institucional y normativo del SNAP. Proyecto Fortalecimiento del Proceso de Implementación del Sistema Nacional de Áreas Protegidas. Montevideo, Uruguay.

SNAP. (2014). Tabla de superficies del SNAP. Recuperado el 10 de 03 de 2015, de SNAP: http:// www.mvotma.gub.uy/snap

SNAP. (2015). SNAP. Recuperado el 01 de 10 de 2015, de Sistema Nacional de Áreas Protegidas de Cuba: http://www.snap.cu/ 
Soutullo, A., Clavijo, C., \& Martínez-Lanfranco, J. (2013). Especies prioritarias para la conservación en Uruguay. Vertebrados, moluscos continentales y plantas vasculares. Montevideo, Uruguay.: SNAP/DINAMA/MVOTMA y DICYT/ MEC.

Tejera, R. (2006). La política de áreas protegidas en el Uruguay (1993 - 2005). . Montevideo, Uruguay.: Universidad de la República.

Vales, M. A., Álvarez, L., Montes, L., \& A., Á. (1998). Estudio nacional sobre la diversidad biológica en la República de Cuba. . La Habana, Cuba: PNUMA; CNB; IES; CITMA.

Cita

Goldberg, N., H. Ferro-Azcona, A. Espinoza-Tenorio, A. Ortega-Argueta, M. A. Mesa-Jurado, E. BarbaMacías. 2016. Sistemas nacionales de áreas protegidas en América Latina; los casos de Cuba,Uruguay y México. Vol.II (1): 63-84. DOI:10.18242/ANPScripta.2015.02.02.01.0005

Sometido: 05 de enero de 2016

Revisado: 18 de marzo de 2016

Aceptado: 02 de mayo de 2016

Editor asociado: Dr. Alfredo Ortega-Rubio

Idioma Inglés Abstract: Ms.C. Diana Dorantes

Diseño gráfico editorial: Lic. Gerardo Hernández 